\title{
KALIDEOS OSR MIPY : UN OBSERVATOIRE POUR LA RECHERCHE ET LA DEMONSTRATION DES APPLICATIONS DE LA TELEDETECTION A LA GESTION DES TERRITOIRES
}

\author{
Jean-François Dejoux, Gérard Dedieu, Olivier Hagolle, Danièle Ducrot, Jean-Claude Menaut, Eric Ceschia, \\ Frédéric Baup, Valérie Demarez, Claire Marais-Sicre, Mohamed Kadiri, Simon Gascoin \\ CESBIO - Centre d'Etudes Spatiales de la BIOsphère \\ Unite mixte CNES-CNRS-UPS-IRD- UMR 5126 \\ BPI 2801 - 18 AVENUE EDOUARD BELIN \\ 31401 TOULOUSE CEDEX 9 - FRANCE \\ Adresse physique du CESBIO : 13, avenue du colonel Roche, Toulouse \\ Tel : (33) 561558597 \\ E-mail : jean-francois.dejoux@cesbio.cnes.fr
}

\section{Résumé}

Ces dernières années, le CESBIO a mis en place un Observatoire Spatial Régional 'OSR' : un dispositif d'observation couplant mesures de terrain et télédétection dans le sud-ouest de la France. L'OSR se base sur des acquisitions mensuelles de données satellitaires à résolution décamétrique depuis 2002 et sur des sites expérimentaux lourdement instrumentés (mesures en continu de flux d'eau et de carbone) à partir de 2004. Ce dispositif a été reconnu service d'observation par l'INSU/CNRS en 2007 et site KALIDEOS par le CNES fin 2009 : 'KALIDEOS OSR MiPy'. Le site atelier correspond à une emprise d'image SPOT, soit environ $50 \times 50 \mathrm{~km}$ et couvre une grande diversité de milieux (pédologie, topographie), d'occupation et d'utilisation des sols, de pratiques et de modalités de gestion (agricole, forestière...) et de conditions climatiques (fort gradient de déficits hydriques estivaux).

Pour la télédétection, ce site a servi la préparation de SMOS, et il soutient maintenant en priorité à la préparation des missions VEN $\mu$ S et Sentinel-2. Les aspects radar, imagerie thermique et les approches multi-capteurs se développent depuis peu. Le traitement du signal, la physique de la mesure et l'amélioration de la qualité des données constituent le premier axe de recherche. Au niveau thématique, le CESBIO a pour priorité les suivis et les modélisations des agrosystèmes de grandes cultures. L'implication récente d'autres partenaires scientifiques ou gestionnaires a permis d'initier des travaux sur d'autres aspects, comme la biodiversité, l'aménagement du territoire, le suivi de l'extension urbaine, les risques environnementaux, la santé des forêts, l'enfrichement, la diversité et la productivité des prairies. La valorisation des 10 années d'archives 2002-2011 débute et semble très pertinente pour la caractérisation en haute et en basse résolution des conséquences d'années climatiques atypiques (2003, 2011) sur les éco-agro-systèmes. L'extrapolation des résultats obtenus sur ce site atelier à toute la région Midi-Pyrénées ou à la chaine des Pyrénées est aussi initiée.

Mots-clés : observatoire, long terme, modélisation, occupation du sol, validation, séries multi-temporelles.

\section{Abstract}

For the last 10 years, CESBIO has been building a Regional Spatial Observatory (OSR), dedicated to coupled terrain and space observations, in the South-West of France. The OSR relies on monthly acquisition of optical images with a 10 meter resolution, started in 2002, and on heavy instrumented sites layed out since 2004 (permanent measurements of water and carbon fluxes). This system was labeled "observation service" by CNRS/INSU in 2007, and became a CNES KALIDEOS site by the end of 2009. The core site has the extent of a SPOT image (50*50km) and spans over very diverse landscapes, with large variations in pedology and topography and diverse land covers, land uses, agricultural practices and climates (with a strong gradient in summer water stress).

Regarding remote sensing, this site has served the preparation of SMOS, and it is now mainly used to anticipate the launch of VEN $\mu$ S and Sentinel-2 satellite missions. Radar and thermal image use is growing and combined approaches are being developed. The research focuses on image quality enhancement, quantitative measurements and signal processing. Regarding thematic research, CESBIO focuses on cereal and oleaginous crops modeling and monitoring. The involvement of new scientific partners and of local managers prompted the development of new studies such as biodiversity, land management, and urban extension monitoring, environmental risks, forest health, grassland diversity and productivity. CESBIO and partners are also starting new studies to make the most of a 10 years archive of data: this archive seems really relevant to study the impact of the atypical climates observed in 2003 and 2011. Finally, the extrapolation to the whole region Midi-Pyrénées or to the whole Pyrenees Mountains has started.

Keywords: observatory, long-term, modelling, land cover, validation, time series. 


\section{Introduction}

Il existe un besoin croissant de connaissances et d'outils pour suivre le fonctionnement des espaces ruraux, aider à mieux gérer les ressources naturelles et anticiper les conséquences des changements globaux. L'utilisation combinée d'observations sur le long terme, de données de télédétection et de modèles constitue un outil indispensable pour aborder ces questions. Le CESBIO s'est spécialisé sur ces problématiques et outils, aux échelles dites "intermédiaires" qui recouvrent celles de l'écosystème, du paysage, du bassin versant et de la région. Ces échelles sont en effet pertinentes pour l'action et la décision, mais elles sont peu étudiées, les approches à la parcelle (agronomie...) ou continentales (télédétection) ayant été plus investies.

Deux chantiers de recherche inscrits dans la durée ont été mis en place par le CESBIO au début des années 2000, l'un dans le sud-ouest de la France et l'autre au Maroc. Dans le sud-ouest, les acquisitions continues de données satellitaires à résolution décamétrique ont commencé en 2002 et l'installation de sites expérimentaux lourdement instrumentés (mesures en continu de flux d'eau et de carbone) en 2004. Ce dispositif constitue un système d'observation unique en son genre pour les questions de gestion de l'eau et d'évaluation des flux et stocks de carbone aux échelles régionales. Ce concept, nommé OSR (Observatoire Spatial Régional), a été reconnu par l'INSU en 2007 (labellisation Service d'Observation). Ce dispositif a aussi été reconnu et soutenu par le CNES à partir de fin 2009 comme dispositif KALIDEOS (nommé KALIDEOS OSR MiPy).

L'objectif de l'OSR est de collecter, de gérer et de mettre à disposition un ensemble de mesures et d'informations caractérisant le fonctionnement et les évolutions des surfaces continentales aux échelles régionales. L'OSR est destiné à fournir des observations régulières sur de longues périodes à un ensemble d'activités de recherche qui ressortent classiquement de quatre catégories :

- suivi et quantification des évolutions de la couverture végétale en réponse à des pressions climatiques ou anthropiques, diagnostics de l'état des milieux ;

- compréhension du fonctionnement d'un ensemble hétérogène d'écosystèmes constituant un paysage, d'un ensemble de paysages constitutifs d'une région ;

- modélisation du fonctionnement de ces systèmes ;

- scénarios potentiels d'évolution de la structure et du fonctionnement de ces systèmes.

L'OSR est également destiné à l'accompagnement des missions spatiales, depuis l'émergence du concept de mission et la distribution de produits de démonstration, jusqu'à la validation des produits et leur valorisation. Un troisième objectif concerne le transfert et la valorisation des travaux de recherche sur le thème de la gestion durable des territoires et de leurs ressources et la sensibilisation des acteurs et du grand public.

\section{Site d'étude et données disponibles}

\subsection{Choix du site d'étude, diversité des conditions de milieu et des modes de gestion}

La zone géographique concernée par l'OSR MiPy est l'ensemble du bassin Adour-Garonne, abordée au moyen d'une approche emboîtée. Deux niveaux géographiques sont pris en compte :

- un site atelier d'environ 50x50 km situé en MidiPyrénées, au sud-ouest de Toulouse. Sur ce site se concentrent les mesures in situ et les mesures de télédétection spatiale à haute résolution ;

- l'ensemble du bassin Adour-Garonne et l'ensemble de la chaine des Pyrénées, abordés dans un premier temps à l'aide de mesures de télédétection à moyenne et basse résolution, ou, plus récemment, pour certaines années, par des séries LANDSAT TM.

Les projets de recherche du CESBIO dans le sud-ouest de la France concernent le suivi et la modélisation des flux et bilans d'eau et de carbone, avec une priorité sur les systèmes de grandes cultures ; les prairies, les forêts et les autres milieux naturels seront intégrés aux bilans régionaux dans un second temps.

Le site atelier a été choisi en 2001 avec plusieurs objectifs: (i) couvrir une grande diversité de milieux (pédologie, topographie, climat), d'occupation et d'utilisation des sols, de pratiques et de modalités de gestion (agricole, forestière...), (ii) permettre un suivi en continu sur au moins une décennie, (iii) obtenir suffisamment de diversité pour l'évaluation des produits de télédétection ou des modèles de fonctionnement. En effet, par rapport à la validation classique, assez ponctuelle dans le temps et dans l'espace, qui peut laisser des marges d'incertitudes relativement importantes, l'observation couplée sol-espace à long terme et sur des surfaces hétérogènes procure une validation qui tient compte de la variabilité spatiotemporelle naturelle des systèmes observés.

Outre la diversité recherchée de milieux et d'activités humaines, ce site est facile d'accès pour les chercheurs basés à Toulouse, et déjà documenté. De grands changements dans l'utilisation des terres y ont été observés depuis 50 ans.

Le site atelier est situé dans les $2500 \mathrm{~km}^{2}$ de l'emprise de référence d'une image SPOT où alternent des vallées (plaines alluviales de la Garonne, de la Save...), des 
terrasses limoneuses associées et des coteaux argilocalcaires (coteaux de Gascogne) (Figure 1). Les deux principaux systèmes de production céréaliers coexistent sur ce territoire : les systèmes basés sur l'irrigation des cultures d'été tels le maïs ou le soja et les systèmes non irrigués où prédominent les rotations à base de blé dur et de tournesol.

Deux grands types de sols sont présents, en lien avec la topographie. Les plaines alluviales et les terrasses associées sont des sols du quaternaire. Une part importante de ces sols sont initialement de mauvaise qualité, car ce sont des sols lessivés en argile et calcium ; des couches imperméables sont présentes à profondeur variable, d'où des problèmes d'hydromorphie en hiver, et parfois de faibles réserves utiles. Ces sols limoneux sont en conséquence très battants, froids au printemps.

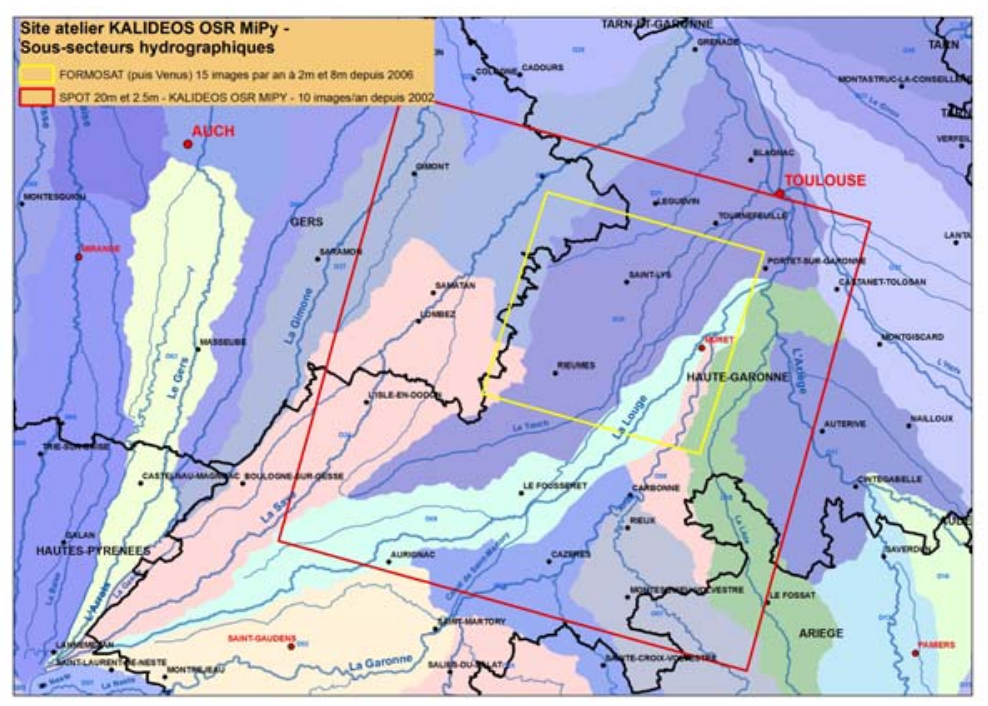

Figure 1 : Site atelier KALIDEOS OSR MIPY.

Les interactions entre ces sols, le climat, les pratiques agricoles et les conséquences sur l'état des cultures (périodes de travail du sol, période de levée, vitesse de croissance, réserves en eau des sols...) sont extrêmement nombreuses, ce qui offre des possibilités très riches de test des modèles.

Initialement, les prairies permanentes étaient nombreuses dans ces zones. Au cours des années 60, 70 et 80 , des changements radicaux ont transformé ces paysages et les pratiques agricoles: des drainages ont été effectués sur des surfaces considérables, l'irrigation a été développée, les surfaces de maïs et soja irrigués et à haut rendement ont remplacé les surfaces en herbe peu productives.

Un autre type de situation domine dans la zone étudiée : ce sont les coteaux argilo-calcaires (Lauragais, coteaux de Gascogne...). Les sols sont développés sur des substrats du secondaire (des molasses érodées des Pyrénées), nommés localement les 'terreforts'; ces sols sont en général assez fertiles ; leur productivité dépend surtout de leur profondeur et donc de leur réserve utile, qui est très variable, et est elle-même dépendante de caractéristiques locales de topographie. L'irrigation est très rare, sauf si des retenues collinaires ont été créées, les systèmes de culture se sont spécialisés autour du tournesol, des blés durs ou tendres et du colza.

L'agrandissement des parcelles au cours des 30 à 50 dernières années a accru les risques de pollutions (azote, phytosanitaires) et d'érosion. La forte proportion de tournesols dans les rotations, qui conduit à laisser les sols nus plus de 8 mois avant l'implantation des tournesols, et la fréquence de sols pentus accentuent aussi ces risques.

Pour les deux situations pédologiques présentées, la variabilité des conditions de croissance est très importante, entre parcelles voisines comme en intraparcellaire, ce qui en fait une difficulté méthodologique (échantillonnage, caractérisation des sols...) mais aussi renforce l'intérêt du suivi par télédétection à des résolutions de 10 à 20 mètres.

D'autres occupations du sol, aux modes de gestion assez contrastés, sont bien représentés dans l'OSR MiPy, comme les différents types de forêts (taillis seuls ou sous futaies de chênes, plantations de résineux, de peupliers, d'eucalyptus...), un gradient de prairies, intensives à extensives, des zones en abandon (enfrichement) surtout sur les zones de pentes, et au contraire, des zones sous forte pression d'urbanisation, en périphérie de Toulouse.

Un dernier intérêt de cette zone, et non des moindres pour les travaux scientifiques menés, est le gradient climatique moyen qui existe en moins de $50 \mathrm{~km}$, entre le sud de la zone (région d'Aurignac) et le nord de la zone, près de Toulouse, avec des sols et des occupations du sol parfois similaires. Sur l'ensemble des 6 mois qui correspond à la période active de croissance de la 
végétation (avril à septembre), le déficit en eau, en $\mathrm{mm}$, atteint en moyenne sur la décennie 2000-2010, 300 à $400 \mathrm{~mm}$ près de Toulouse et seulement 50 à $150 \mathrm{~mm}$ près d'Aurignac. Ce gradient est causé par les différences d'altitude (piémont pyrénéen, 300 à 400mètres; plaines autour de Toulouse, 150 mètres) mais surtout par la proximité des Pyrénées qui provoque des pluies plus abondantes dans le piémont. Cette zone est donc très bien adaptée pour l'étude de la réaction des écosystèmes aux conditions climatiques contrastées et changeantes.

\subsection{La composante télédétection de I'OSR MiPy}

L'OSR repose sur la collecte de deux types d'informations :

- mesures de terrain,

- mesures par capteur satellitaire, à diverses résolutions spatiales et temporelles, dans plusieurs domaines de longueurs d'onde ;

Les principaux travaux basés sur le dispositif OSR MiPy concernent les écosystèmes cultivés ou naturels aux échelles régionales, ce qui a deux conséquences sur le type d'images satellites étudié : priorité aux résolutions décamétriques ( 5 à 30 mètres de résolution spatiale) et aux séries multi-temporelles (8 à 30 images par an). In fine, il s'agit de préparer les algorithmes et les outils capables de valoriser les images des futurs satellites GMES-Sentinel-2, les prochains LANDSAT et VEN $\mu S$ en optique et Sentinel-1, TerraSAR-X, RADARSAT-2... en radar: calibration, validation, mise au point des chaines de production thématiques.

La série d'images de référence est constituée par une centaine d'images SPOT de 2002 à 2011, à 20 et 10 mètres de résolution spatiale, soit environ 10 images par an, plus quelques archives de 1991 et 1994. La répétitivité recherchée est de 1 image par mois en moyenne, mais ce besoin est plus fort pendant la période de croissance de la végétation, de mars à septembre, qu'en hiver.

L'utilisation de séries de télédétection pour le suivi de la végétation, dans les outils et modèles de fonctionnement des écosystèmes renforce le besoin d'obtenir des images aussi bien corrigées que possible des perturbations de l'atmosphère, afin que les valeurs de réflectances au sol soient les plus exactes possibles. Pour cela, des travaux récents [Hagolle et al., 2008, Hagolle et al., 2010] ont montré tout l'intérêt des séries multi-temporelles acquises avec le même angle de visée. Plusieurs futures missions spatiales (GMESSentinel-2, les prochains LANDSAT et Venus) auront cette caractéristique. Pour les préparer au mieux, une acquisition systématique d'images FORMOSAT-2 (à 8 mètres de résolution en multi-spectral et 2 mètres en panchromatique) est réalisée depuis 2006, avec 10 à 20 images sans nuages par an. La zone observée est incluse dans la zone SPOT, elle couvre environ $500 \mathrm{~km}^{2}$ contre $2500 \mathrm{~km}^{2}$ pour la zone de référence SPOT. Combinée avec les images SPOT, un suivi très fin dans le temps est donc possible sur cette zone, avec 20 à 25 images SPOT ou FORMOSAT-2 par an bien réparties dans l'année, de 2006 à 2011.

Une autre stratégie pour préparer la future mission Sentinel-2, est d'utiliser les archives LANDSAT TM 5 ou 7. Ces images sont en effet acquises depuis 1985 avec des angles de visée constants, leur qualité et leur résolution (30 mètres) sont moindres que celles de SPOT ou FORMOSAT-2, mais leur recul historique (1985) et leur très large emprise $(180$ * $180 \mathrm{~km}$ par scène) en font des données très complémentaires aux images SPOT et FORMOSAT-2. En 2011, à l'occasion de plusieurs projets, le CESBIO a donc récupéré et traité des archives LANDSAT sur toutes les Pyrénées pour 2 périodes (2002/2003 et 2009/2010) et bientôt une 3ème période (début des années 1990) sous forme de séries multi-temporelles, avec 4 à 8 dates sans nuages par an, ceci afin de réaliser des études diachroniques d'évolution de l'occupation du sol.

La basse résolution a encore été peu valorisée, seule ou en complément de la résolution décamétrique. Le CESBIO et l'Ecole d'Ingénieur de PURPAN commencent certains travaux à ce sujet (anomalies de végétation sur la décennie et caractérisation d'années climatiques particulières comme 2003 ou 2011).

De même, la très haute résolution spatiale, de type Pléiades, a fait l'objet de premiers travaux récemment (depuis 2009), dans le cadre du programme ORFEO du CNES. DYNAFOR, GEODE et CESBIO sont en effet intéressés par les questions de cartographie des trames vertes et bleues et de détection des haies.

Malgré leur précision et leur grande richesse, les données optiques présentent le grave inconvénient de devenir indisponibles lorsqu'une couverture nuageuse s'installe pour de longues périodes. Pour assurer l'obtention de données par tous les temps, la tendance récente du site OSR MiPy est la recherche de complémentarité entre les mesures optiques et le radar, toujours aux échelles compatibles avec la parcelle agricole.

Une campagne spécifique de terrain et d'acquisitions par satellite a été menée en 2010 (http://www.cesbio.upstlse.fr/fr/mcm.html). Cette campagne est centrée sur la caractérisation des états de surface (rugosité, résidus...) et l'humidité de surface des sols. II est souhaité qu'un suivi régulier par images radar soit réalisé dans le cadre de KALIDEOS OSR MiPy (images TerraSAR-X, voire RADARSAT bande $C$ et prochainement Sentinel $1 \mathrm{a}$ et 1b).

Enfin, dans cette zone, la disponibilité et l'utilisation raisonnée de l'eau sont des problèmes cruciaux. Certains utilisateurs de l'OSR investissent donc dans l'utilisation de données dans l'infrarouge thermique pour pouvoir réaliser des bilans détaillés d'évapotranspiration. 
Malheureusement, les données thermiques à haute résolution spatiales sont très rares; les meilleures sont fournies par les instruments ASTER et LANDSAT $(\sim 100 \mathrm{~m})$, mais avec une répétitivité très insuffisante.

\subsection{Le traitement des données satellites optiques}

Les données utilisées dans cet article proviennent pour les images SPOT et FORMOSAT-2 du programme KALIDEOS du CNES, qui acquiert ces données et les met à disposition de la communauté scientifique.

Les modalités de traitement des images optiques dans le cadre de KALIDEOS sont explicitées dans l'article de [Lafrance et al., 2010]. Nous présentons ci-dessous les spécificités de l'OSR MiPy, en particulier le choix de l'image de référence. II s'agit d'une acquisition SPOT-5 à 2,5 m couleur (THX) du 31 août 2009, ortho-rectifiées par IGN Espace. C'est aussi une des images utilisée dans le produit Spot-Image - IGN de couverture de la France en 2009 en SPOT-5 2.5 mètres, qui a aussi comme caractéristique une très bonne superposition avec la BD ortho IGN. Le CESBIO a effectué un contrôle visuel du produit livré sur 100 points de la BD ortho.

Cette image est strictement dépourvue de nuages, voiles, traces d'avion. De plus, cette scène appartient à un segment de 6 scènes consécutives acquises par Spot-Image, soit plus de $250 \mathrm{~km}$ du nord au sud, de Limoges jusqu'à la frontière espagnole dans les Pyrénées au niveau de Luchon. Cet aspect peut être intéressant, car d'autres projets se greffent actuellement sur KALIDEOS OSR MiPy, soit au sud (piémont pyrénéen...) soit dans le reste de la région MidiPyrénées ; il sera ainsi possible d'avoir le même type de conditions d'image de référence.

Dans le sud-ouest de la France, les conditions d'août 2009 correspondent à une situation de canicule et sécheresse. Les parcelles en culture d'hiver sont des sols nus très marqués (pas de végétation, repousses ou adventices), la plupart des prairies sont sèches, les cultures irriguées sont très visibles car en pleine végétation, les cultures d'été non irriguées (comme le tournesol) ont eu des dessèchements très précoces (fin juillet) et apparaissent donc comme sol nu. Le seul inconvénient de ces scènes de référence est qu'elles sont loin du nadir (angle zénithal de visée de $29^{\circ}$ ). L'ensemble de l'archive KALIDEOS (SPOT et FORMOSAT-2) a donc été retraité début 2010 à partir de cette image de référence et livré en projection Lambert 93 France. Si besoin, il sera possible à l'avenir de générer une $2^{\text {ème }}$ image de référence quelques années plus tôt, pour prendre en compte les évolutions du paysage (archive disponible en SPOT-5 à 2.5 mètres le 13 décembre 2004).

\subsection{Données terrain et exogènes de l'OSR MiPy}

En plus des mesures par capteur satellitaire, l'OSR repose sur la collecte de deux types d'informations terrain :
- mesures in situ continues par suivi intensif des flux de carbone, d'eau et d'énergie complétées par des mesures continues ou par échantillonnage de variables du sol et de la végétation sur 3 parcelles lourdement équipées ; à ce suivi continu se rajoutent si nécessaire sur quelques autres parcelles des suivis ponctuels ( 1 à 3 cycles de végétation) et allégés du sol et de la végétation ;

- $\quad$ suivi extensif par enquêtes sur l'occupation et l'usage des terres (travail du sol, sens des rangs, accidents de cultures...) de manière continue chaque année sur plusieurs centaines de parcelles. Des enquêtes ponctuelles sur quelques campagnes sont aussi menées pour des variables spécifiques (haies, états de surface...).

De manière croissante depuis 2008, des données exogènes sont traitées et intégrées dans l'observatoire. Il s'agit de bases de données dont l'accès devient de plus en plus aisé pour la recherche, comme les données météorologiques spatialisées (Safran...), la PAC graphique (déclarations des agriculteurs) et nous espérons bientôt les cartes de l'IFN (Inventaire Forestier National), les déclarations de volumes prélevés collectées par l'Agence de l'Eau, les données cartographiques de I'IGN, en particulier du RGE (BD ortho sur la décennie, MNT au pas de 25 mètres, BD topo...).

De nombreuses données spatialisées sont nécessaires aux forçages et validations des modèles, mais difficiles à obtenir et à qualifier: itinéraires techniques agricoles (dates de semis, variétés, fertilisation azotée, travail du sol, dates et doses d'irrigation...), rendements grain à $0 \%$ d'humidité à la parcelle, qualification des facteurs limitants (maladies, verse...). Plusieurs partenariats se mettent en place actuellement pour pérenniser une collecte fiable de ces informations, sur quelques centaines de parcelles agricoles.

Les cartes sur diverses propriétés des sols (réserves utiles, albédo, fonctions de transferts...) sont partielles. Leur amélioration, au moins sur l'emprise des images FORMOSAT-2 est souhaitée, mais le calendrier et la démarche ne sont pas encore fixés.

\subsection{Principaux utilisateurs de KALIDEOS OSR MiPy}

Le CESBIO est le fondateur du concept et du dispositif OSR et le principal utilisateur. Dès l'origine, il était souhaité que ce dispositif soit le plus ouvert possible et utilisé par d'autres équipes scientifiques et par les acteurs.

$\mathrm{Au}$ niveau scientifique, plusieurs laboratoires de recherche sont intéressés par les produits dérivés de I'OSR : cartes de LAI, de besoins en eau, de productivité des écosystèmes; cartes d'occupation du sol et des états de surfaces (sols nus...). Il s'agit de laboratoires de 
géographie (GEODE), d'écologie du paysage (DYNAFOR), d'écologie générale (EDB, EcoLab), d'économie rurale (LERNA, ODR), d'agronomie (Agir), de météorologie (CNRM). Au sein de la communauté scientifique qui étudie les Surfaces et Interfaces Continentales ('SIC'), l'originalité d'un OSR est sa composante spatiale, qui lui confère un atout majeur dans la puissance d'observation en continu mais aussi un handicap de par la zone contrainte et limitée observée, tant que les capteurs opérationnels grand champ (Sentinel-2) ne sont pas en fonctionnement. C'est pourquoi il est pour l'instant difficile d'attirer d'autres laboratoires et de favoriser les synergies sur un territoire d'étude commun : chaque laboratoire, chaque équipe a ses propres logiques de terrains d'études: contraintes thématiques, ressources limitées, connaissance des acteurs, habitudes de travail, proximité géographique, voire positions concurrentielles. Si la diversité des activités de recherche est une richesse, nous pensons qu'un certain nombre de défis liés aux impacts des changements globaux, aux adaptations et au développement durable nécessite le développement de recherches plus intégrées et pluridisciplinaires sur un territoire commun et associant la composante spatiale.

Les liens avec les acteurs sont très nombreux et s'accroissent chaque année. Ces liens s'organisent sous forme de partenariats inscrits si possible dans la durée. La nature des échanges est variée: définition des besoins et des problématiques, collecte de données, retour sur l'utilisation des produits, information sur les méthodologies applicables... Ces acteurs sont principalement les administrations et gestionnaires (service de l'Etat, collectivités, agence de bassin), les organismes professionnels (chambres consulaires, instituts techniques...), les acteurs eux-mêmes (agriculteurs), les bureaux d'études et les industriels.

Plusieurs dispositifs originaux sont développés et structurent les actions en partenariat, comme les Living Labs, destinés à co-construire les besoins et les applications avec les acteurs, et à l'avenir les Territoires Démonstrateurs de Services.

La formation est un aspect important du dispositif. L'OSR est ainsi étroitement articulé avec le master ATT (Aménagement du Territoire et Télédétection) de I'Université Toulouse III et contribue aux travaux de stage d'étudiants issus de nombreuses autres formations.

\section{KALIDEOS OSR MiPy : quelques résultats et applications}

Plusieurs laboratoires de Midi-Pyrénées et d'Aquitaine sont impliqués dans la préparation et la valorisation des missions spatiales à venir (CNRM, INRA-Ephyse, LEGOS, CESBIO). Le CESBIO assure la responsabilité scientifique de trois missions spatiales (SMOS (2009), VEN $\mu S$ (2013), BIOMASS), est très impliqué dans la mission Mistigri, dans les activités préparatoires à l'arrivée de Sentinel-2 (horizon 2014) et Sentinel 1a et $1 b$, et dans les travaux de définition de la suite de SMOS. Ces missions offrent dans plusieurs domaines spectraux des résolutions spatiales et surtout temporelles accrues (e.g. Pléiades, VEN $\mu S$, Sentinel-2, TerraSAR-X, COSMO-SkyMed) ou des mesures nouvelles (SMOS). Le dispositif et service OSR ont tous les atouts pour permettre le développement de méthodologies d'exploitation de ces missions. Une illustration des travaux scientifiques et des premières applications sont détaillées ci-dessous en fonction de trois thèmes.

\subsection{Physique de la mesure et amélioration de la qualité des données}

Dès que l'on s'intéresse à des séries temporelles d'images, l'homogénéité des données à l'intérieur de ces séries devient un critère de qualité primordial. Plusieurs phénomènes viennent nuire à cette homogénéité :

- Les différences entre instruments. Bien que chaque satellite optique ait au moins une bande rouge et une bande proche-infra-rouge, d'importantes différences dans la position et la réponse spectrales peuvent causer des différences dans les réflectances mesurées et les indices de végétation qui en dérivent. Dans certains cas, ces différences ne peuvent pas être négligées. Par ailleurs, le CNES porte une attention soutenue aux différences d'étalonnage entre capteurs, ce qui assure une bonne homogénéité aux données issues de KALIDEOS, pour l'utilisation combinée de SPOT et FORMOSAT-2 par exemple [Cabot et al., 1999].

- Les effets directionnels: Iorsqu'on observe une même cible sous des angles de visée et des angles d'éclairement variables, il est fréquent que la réflectance apparente change. C'est ce qu'on appelle les effets directionnels. Les effets directionnels sont particulièrement importants au dessus de la végétation: Iorsqu'on observe celle-ci avec le soleil dans le dos, seules les faces ensoleillées des feuilles et du sol sont visibles. Lorsque cette observation est faite face au soleil, une partie des feuilles et du sol est à l'ombre. Cet effet peut entraîner des variations d'un facteur 2 des réflectances. Pour éviter cela, les observations du satellite VEN $\mu S$ [Dedieu et al., 2006] seront réalisées à angle constant, alors que les angles d'observation offerts par SPOT peuvent varier de plus de 50 degrés d'une image à l'autre $\left(+/-27^{\circ}\right)$. Pour cette même raison, les données du satellite FORMOSAT-2 utilisées sur l'OSR sont programmées avec des angles constants.

- Les nuages et les effets atmosphériques. D'un jour à l'autre, la transparence de l'atmosphère peut varier très fortement : c'est bien sûr le cas en présence de nuages, mais aussi lorsque le contenu atmosphérique en aérosols varie. Or ce contenu est extrêmement variable, et les variations des réflectances induites 
par les aérosols peuvent atteindre un facteur 2 sur des cibles sombres. A partir des données FORMOSAT-2, et en prévision de l'arrivée de VEN $\mu S$ et de Sentinel-2, une méthode multi-temporelle de détection des nuages et de correction des effets atmosphérique a été mise en place, qui permet de bien mieux détecter les nuages et d'améliorer la qualité des séries temporelles [Hagolle et al., 2008 ; Hagolle et al., 2010].

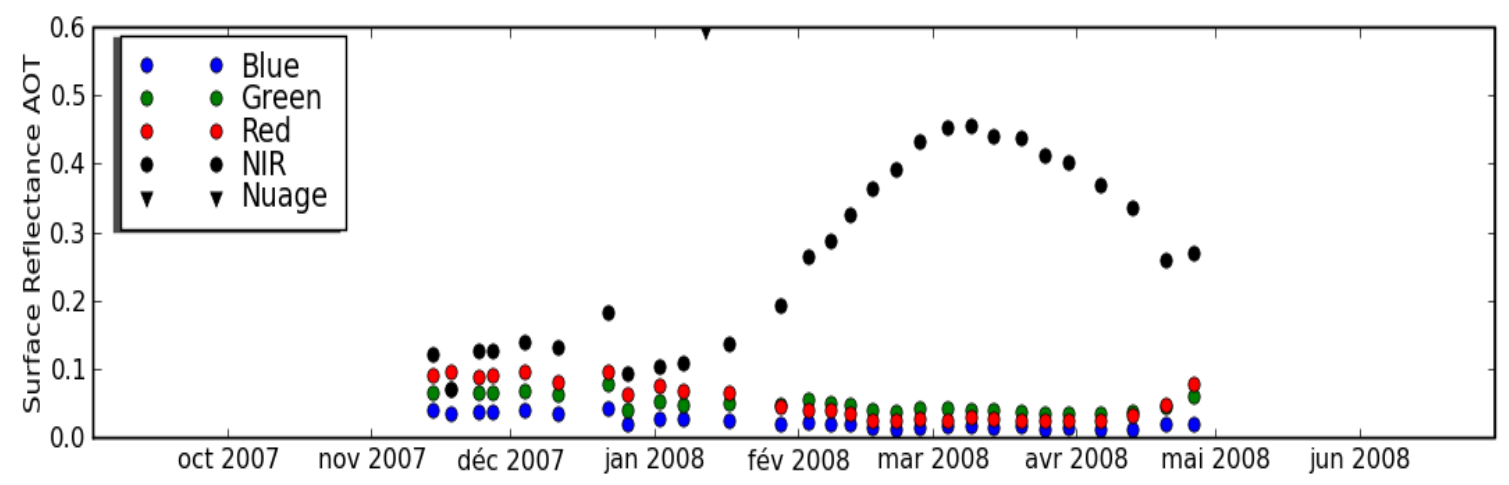

Figure 2 : Suivi en fonction du temps des réflectances d'une parcelle de blé (au Mexique), avec FORMOSAT-2 (acquisitions avec angles constants) et après correction atmosphérique.

\subsection{Cartographie de l'occupation et de l'usage des terres}

La production et la mise à jour des cartes d'occupation du sol sont un enjeu majeur, car elles sont utiles à 2 niveaux. Premièrement, le suivi de l'évolution des occupations du sol est un enjeu scientifique et appliqué majeur pour de nombreuses problématiques : diagnostic des causes d'évolution, associant sciences humaines et étude du milieu, simulation des conséquences des changements sur différents aspects agroenvironnementaux comme le stockage du carbone dans les sols, la biodiversité, les risques d'érosion... Deuxièmement, les cartes d'occupation des sols sont utilisées comme données d'entrée pour la plupart des modèles spatialisés qui estiment différentes variables biophysiques, flux et stocks de matières.

Seuls les travaux de valorisation des séries temporelles d'images décamétriques sont présentés ci-dessous (préparation Sentinel-2), mais d'autres travaux sont menés par le CESBIO, DYNAFOR et GEODE dans le cadre d'ORFEO, préparation de Pléiades, sur les trames vertes et bleues.

Aux échelles régionales, les données actuellement utilisées ou disponibles sont soit des cartes d'occupation du sol à basse résolution, soit des cartes issues de photo-interprétation comme Corine Land Cover 'CLC'. Toutes ces cartes sont en général peu adaptées pour les gestionnaires comme pour les simulations aux échelles du paysage, car à maille trop lâche (25 ha pour CLC 1990, 2000 et 2006; 250 à 1000 mètres de résolution pour ECOCLIMAP, MODIS, MERIS) et trop imprécises.

D'autres bases de données peuvent être très utiles, comme la PAC graphique ('RPG'), disponible depuis 2006 en France ou les inventaires forestiers nationaux
(IFN, maille de 2.25ha et bientôt de 0.5 ha). Le RPG est utilisé de manière intensive au CESBIO, comme données d'apprentissage des classifications supervisées ou en validation. Ces bases de données ont cependant quelques défauts, comme leur non exhaustivité, les délais de production, leur faible fréquence de mise à jour, le besoin de méthodes manuelles d'extraction de l'information, etc...

C'est dans ce contexte que la télédétection peut apporter des réponses, seule ou en complément des données existantes. Aux échelles régionales, la précision demandée, qui dépend de la taille du parcellaire des objets, se satisfait dans le sud-ouest de résolutions comprises entre 10 et 30 mètres pour la plupart des couverts (grands cultures, parcelles forestières, prairies, friches...). L'intérêt majeur de la télédétection, par rapport aux photos aériennes par exemple, repose sur sa répétitivité : c'est la possibilité d'avoir des images (décamétriques) à des saisons différentes qui permet de limiter une part importante des confusions de classification [Ducrot et al., 2010 ; Masse et al., en révision].

Grâce au grand nombre de dates acquises, l'évolution des réponses spectrales est étudiée à partir des valeurs moyennes des réflectances de chaque classe dans chaque canal spectral ou NDVI. Ceci permet de déterminer diverses propriétés des cultures, notamment leur cycle (Figure 4). L'évolution temporelle des courbes de réflectance informe sur les différentes stades phénologiques des cultures: semis, croissance, sénescence, récolte, et les phases d'intercultures.

Parmi les travaux menés au CESBIO par Danielle Ducrot depuis quelques années, en classification supervisée ou non supervisée, un des sujets est de déterminer le meilleur compromis entre nombre et dates des images et performance des classifications (Figures 3 et 5 ). Suivant les classes, les meilleures combinaisons 
ne sont pas toujours les mêmes. Ces résultats prouvent bien qu'avoir un résultat de classification supervisée excellent n'est pas immédiat et que ces résultats sont très dépendants de l'échantillonnage. Les résultats dans les graphiques ci-après donnent les pourcentages de bien classés globaux MPCC (/Moyenne de pourcentages de correctement classés ou Average Accuracy/) avec les dates ajoutées chronologiquement. Ils sont présentés à partir de l'échantillonnage complet d'une part et avec deux jeux d'échantillons différents pour l'apprentissage et la vérification. On peut ainsi observer l'apport du multitemporel. Des graphiques de pourcentage de correctement classés (PCC) par type de cultures sont également donnés, ce qui permet d'étudier les canaux spectraux et temporels pour la détection optimale des classes. La Figure 5 illustre le PCC pour du blé.

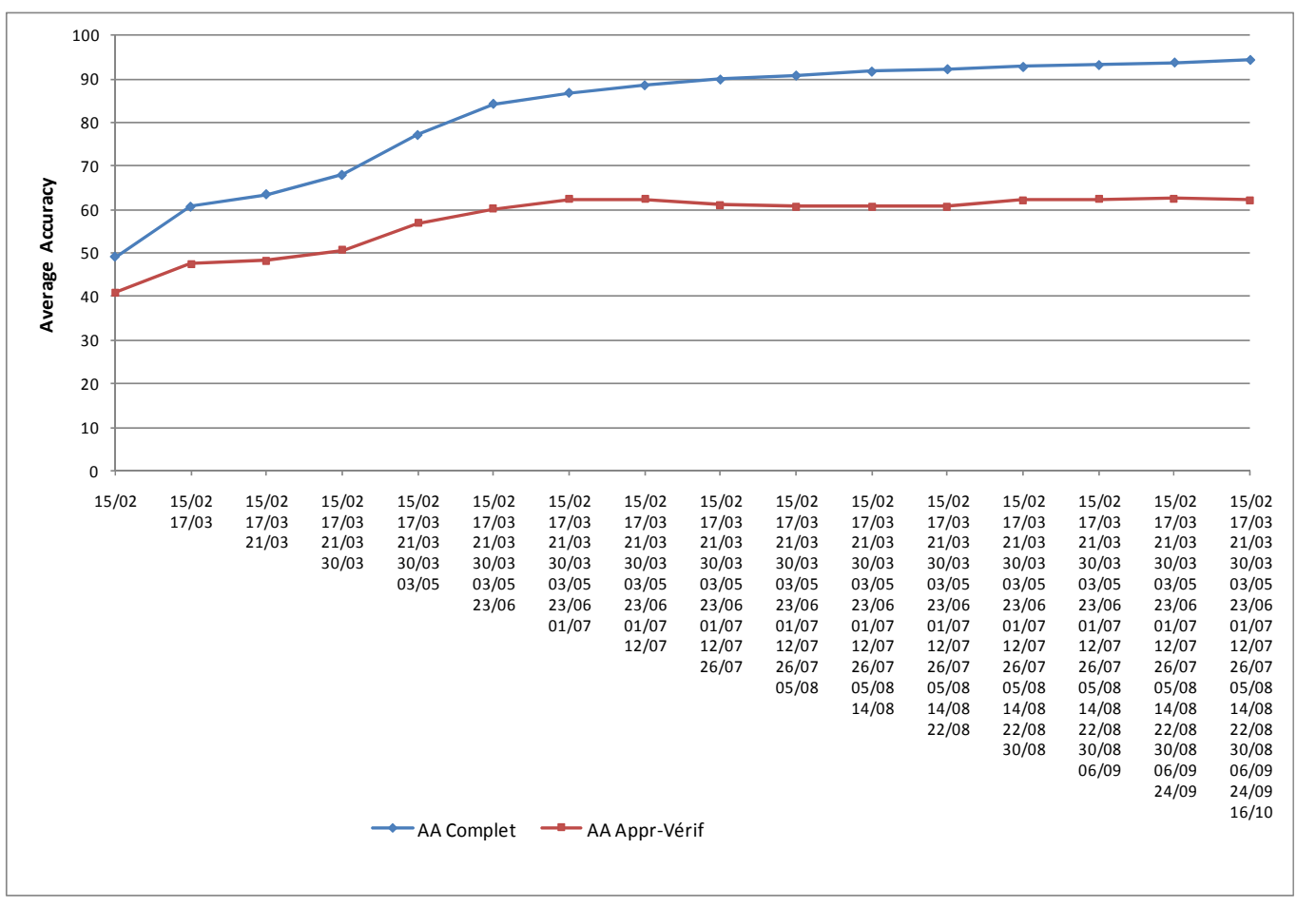

Figure 3 : Comparaison du MPCC (Moyenne des Pixels Correctement Classées ou Average Accuracy) en addition chronologique des 16 dates de 2009 avec les jeux d'échantillons complet et apprentissage-vérification

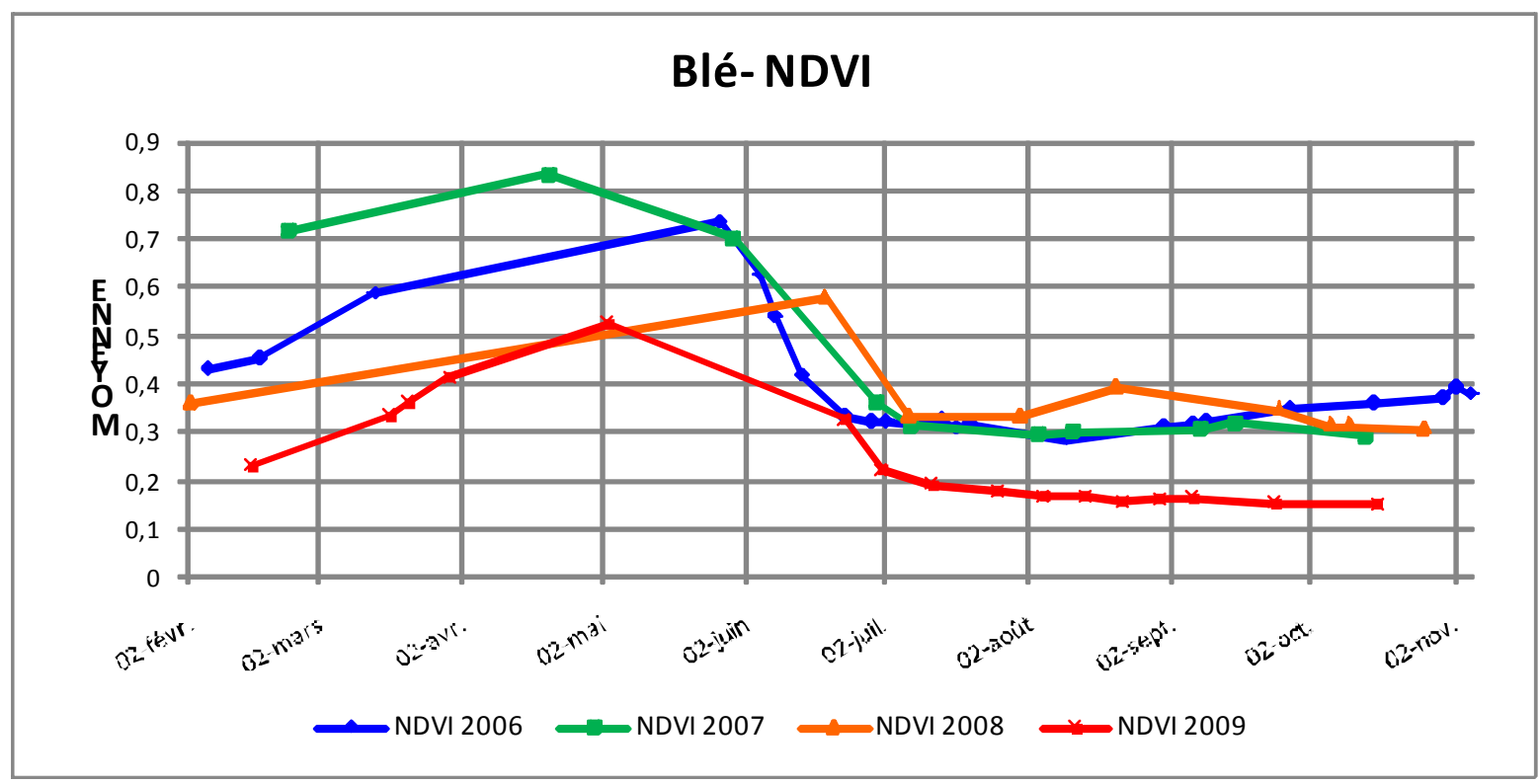

Figure 4 : Comparaison des réponses spectrales de la classe Blé dans le NDVI pour les années 2006-2007-2008-2009. 


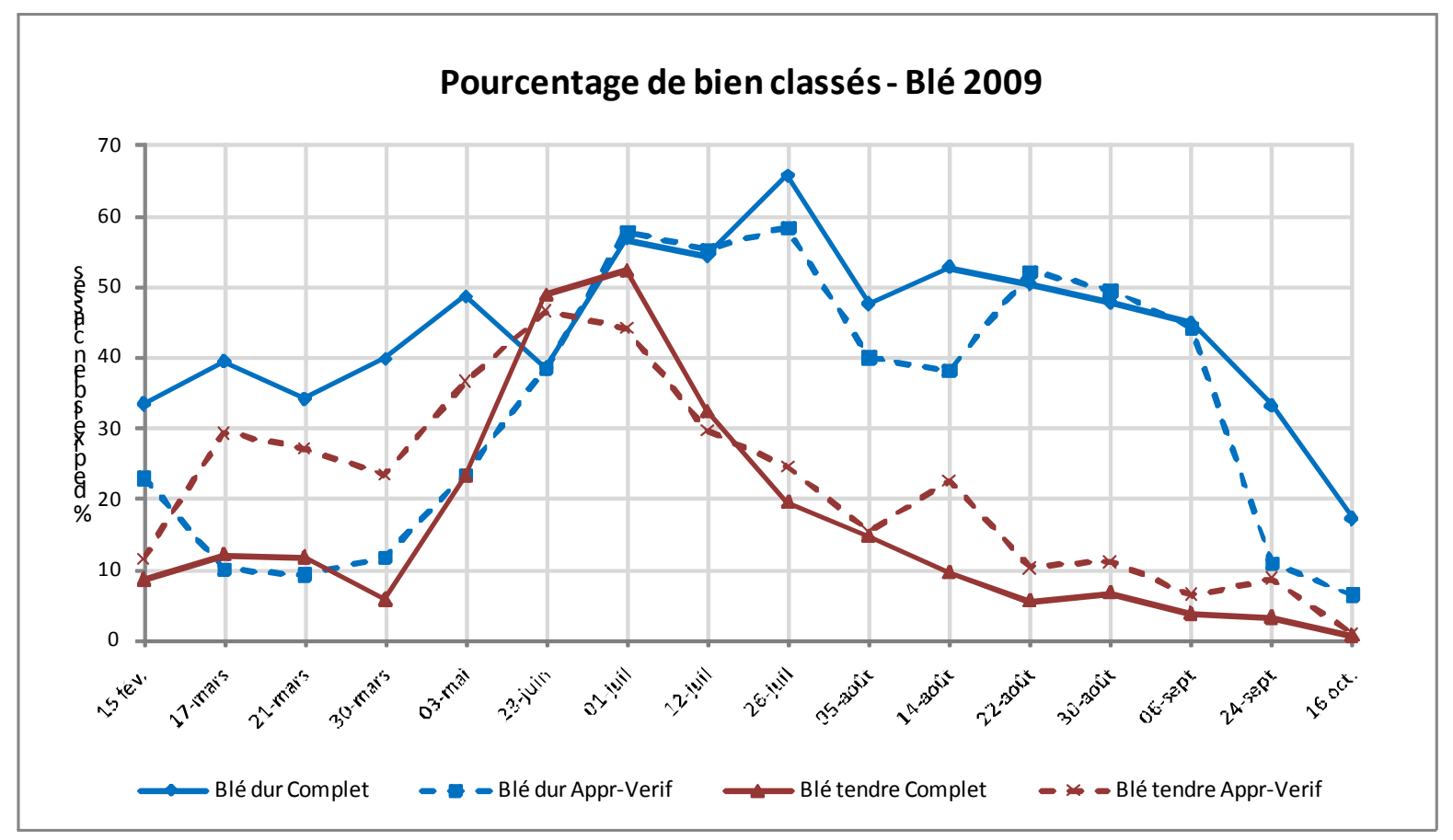

Figure 5 : Pourcentage des pixels de Blé bien classés $(P C C)$ avec une seule date.

\subsection{Valorisation de la diversité climatique de l'observatoire et classification fonctionnelle}

Un des intérêts d'un observatoire spatial est le suivi continu de milliers de parcelles sur une longue durée. Associé à la modélisation, ce suivi doit d'une part permettre d'observer la réaction des agro écosystèmes aux différentes années climatiques: évènements rares (sécheresse ou canicule exceptionnelles...), effets cumulés, puis d'autre part être à la base de démarches de simulations des flux et bilan de Carbone et d'eau sous différents scenarii de changement globaux.

Le recul historique du suivi par télédétection de l'OSR est actuellement de 10 années (2002 à 2011 inclus). ॥ s'avère que la diversité des années climatiques de cette décennie est exceptionnellement riche dans le sudouest, d'où l'intérêt de mener des analyses de la réaction des écosystèmes à ces conditions climatiques contrastées, qui peuvent être résumées ainsi pour les $6 / 8$ mois de période de végétation (février/mars à octobre/novembre) :

- Années normales, voire fraiches et humides en été : 2002, 2004, 2007 et 2008.

- Printemps chauds et secs : 2003, 2005, 2006, 2011.

- Etés et début d'automne chauds et secs : 2003, 2005, 2006, 2009, 2010.

Comme cela a été souligné par Météo-France au niveau national, un enchainement de plusieurs années particulières s'observe de 2003 à 2006 ; mais la période 2009 à 2011 semble aussi constituer un enchainement de conditions chaudes et sèches sur la plupart des mois du printemps à l'automne. La question des effets cumulatifs sur la végétation est à évaluer.

L'archive KALIDEOS OSR MIPY a été retraitée et livrée au printemps 2011, ce qui permet les premières analyses, basées sur les corrélations entre dynamiques de NDVI et conditions météorologiques et mise en évidence des anomalies de NDVI. L'impact de la sécheresse 2003 avait déjà été valorisée par [Coret et al., 2005], c'est pourquoi nous avons mis à jour ces analyses jusqu'aux données les plus récentes de 2011. Sur la Figure 6 est représentée le NDVI de 90 parcelles de prairies permanentes (surface moyenne de 6 ha avant érosion puis de 3 ha après érosion) de la zone FORMOSAT-2 de KALIDEOS OSR MIPY. Ces parcelles ont été sélectionnées à partir du RPG 2006 à 2010, nous avons donc fait l'hypothèse que ces parcelles étaient déjà en prairies permanentes les 4 années précédentes (2002-2006). Toutes les images FORMOSAT-2 et SPOT de l'archive KALIDEOS ont été utilisées, après ré-échantillonnage bicubique à 8 mètres de résolutions des images SPOT, soit 146 dates d'avril 2002 au 21 mai 2011. Les dynamiques de NDVI sont très cohérentes avec les conditions climatiques, par exemple, les chutes associés aux printemps chauds et secs $(2003,2006,2011)$, aux étés chauds et secs (2003, 2005, 2006), aux fins d'été et automnes chauds et secs (2003, 2009, 2010). A contrario, on observe les dynamiques de croissance de fin d'été et d'automne très fortes en 2005 et 2006, dues à la combinaison de pluies de fin d'été (aout 2005 ou septembre 2006) et de températures anormalement douces en octobre 2005, ou de septembre 2006 à février 2007. Seule l'année 2004 est mal corrélée aux conditions météorologiques, le NDVI est anormalement faible au printemps et en été. 


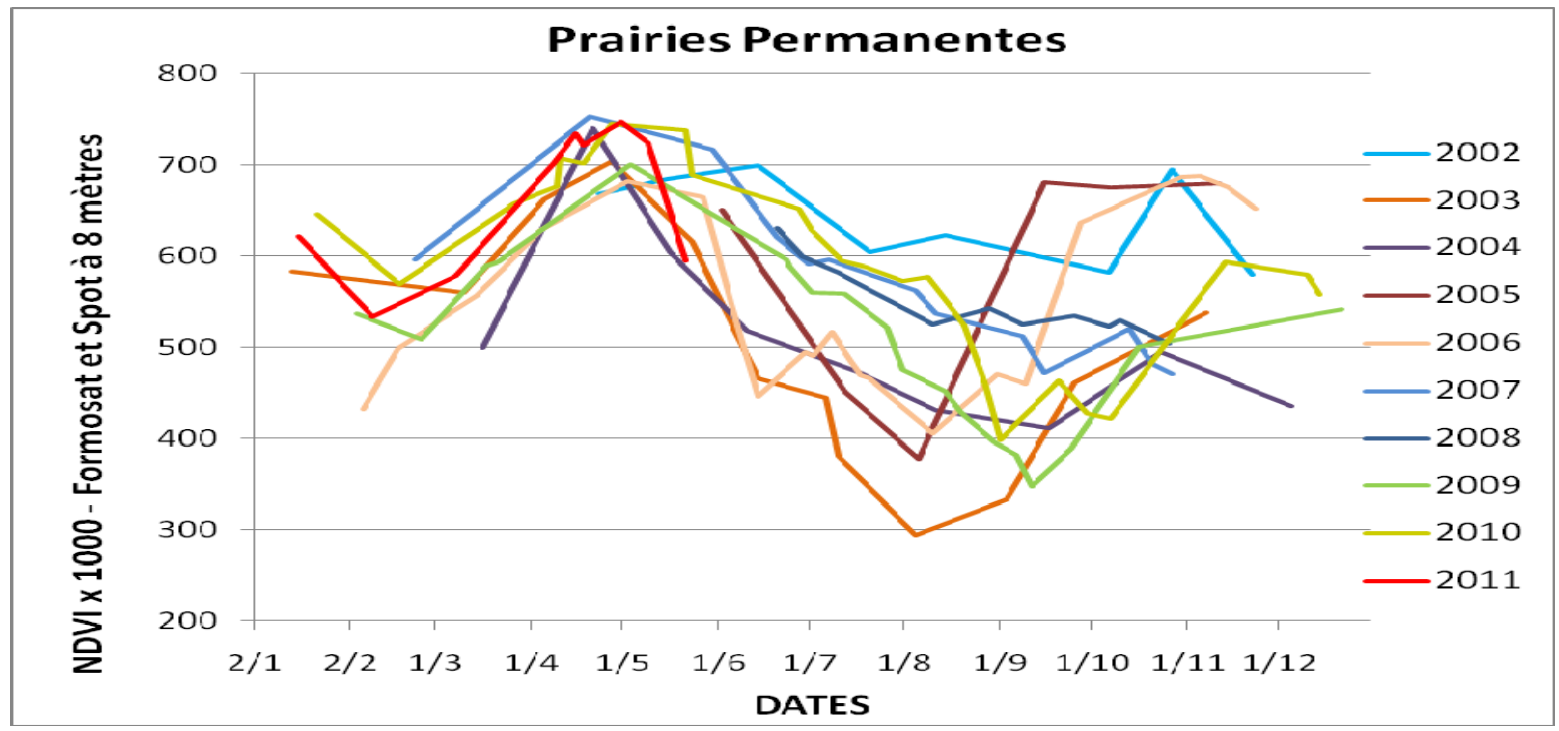

Figure 6 : NDVI moyen sur 90 parcelles de prairies permanentes (source RPG 2006-2010) pour 146 dates SPOT et FORMOSAT-2 à 8 mètres de résolution.

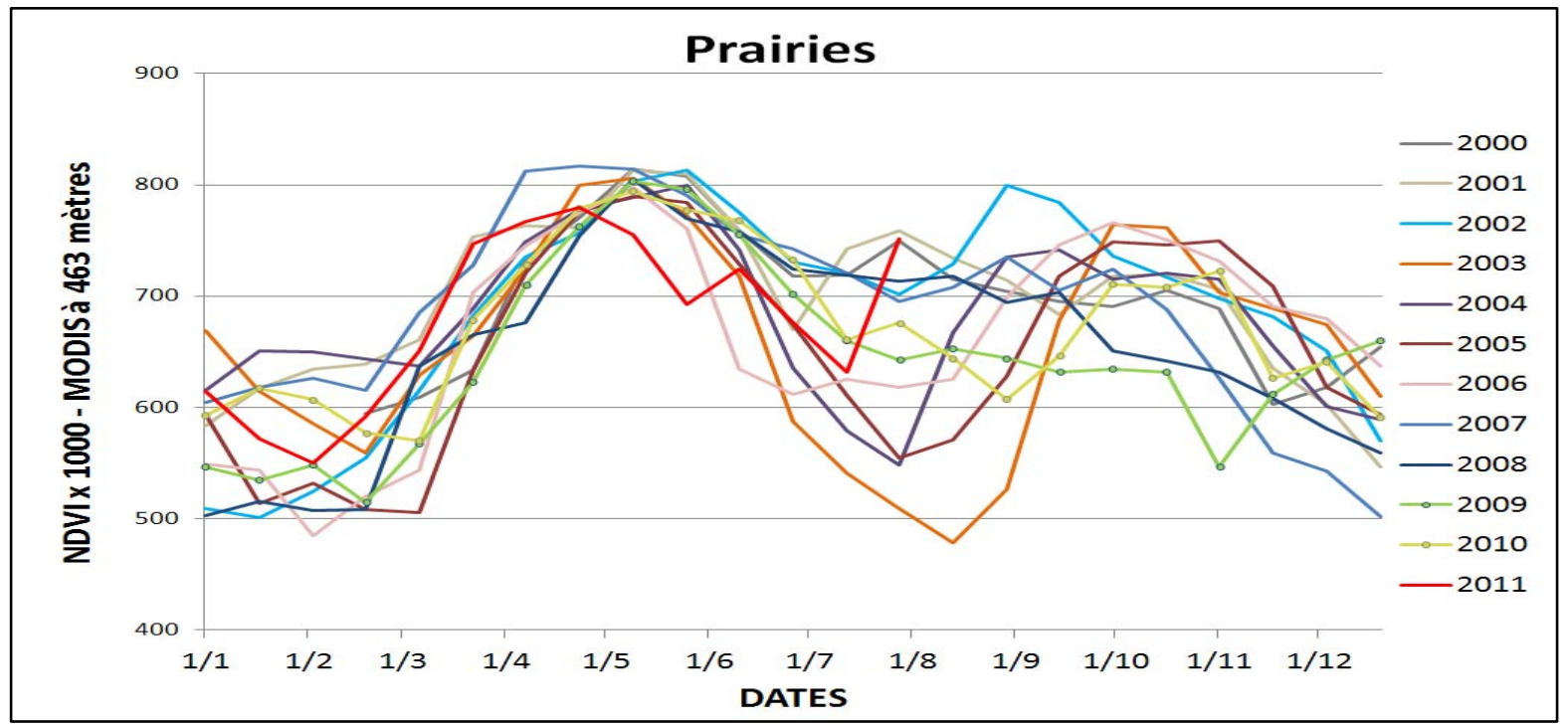

Figure 7 : NDVI moyen de pixels MODIS purs selon Corine Land Cover, classe 231 'Prairies', sélectionnés sur la région MidiPyrénées.

La fréquence des images SPOT ou FORMOSAT-2 étant limitée, il est intéressant d'avoir une référence temporelle plus fine, avec des capteurs à basse résolution, qui présentent aussi l'avantage d'échantillonner de plus grandes zones. Un travail en cours caractérise les dynamiques de NDVI pour l'ensemble de la région Midi-Pyrénées à partir de synthèse MODIS à 16 jours et 500 mètres de résolution (Gascoin, Dejoux, Kadiri, Figure 7).Des pixels Modis avec un seul type d'occupation du sol ont été sélectionnés à partir des cartes d'occupation du sol Corine Land Cover 2006 (CLC) afin d'obtenir un signal relativement homogène par groupes d'espèces. Seuls les aspects prairies sont présentés ci-dessous et comparés aux suivis à haute résolution spatiale. La classe 231 de CLC 'prairies' est présente dans toute la région sauf en altitude, elle correspond principalement à des parcelles de prairies permanentes, et inclut parfois des prairies temporaires. On observe des dynamiques NDVI très cohérentes avec les conditions climatiques. L'année 2011 semble battre des records, même comparé à 2003 , en termes de précocité de croissance en avril, de chute de NDVI en mai, et enfin de réaugmentation du NDVI en juillet. Cette démarche qui 
combine la haute et la basse résolution spatiale fait actuellement l'objet d'une collaboration entre l'école d'ingénieur de PURPAN et le CESBIO et sera valorisée dans une thèse menée à PURPAN sur les risques de dépérissement des chênes pédonculés et de quelques autres essences forestières, en lien avec les changements climatiques.

Ces analyses de comportement de classes de végétation supposent en amont que les cartes d'occupation du sol soient disponibles, précises et fiables. Dans notre travail, nous utilisons à cet effet des extraits des bases de données RPG ou IFN. Dans une deuxième étape, il est nécessaire de contrôler la fiabilité de ces bases, et repérer toutes les erreurs. Ensuite, des analyses plus fonctionnelles sont possibles, par des approches de classifications intra-classes. Selon les résultats obtenus, il est alors possible de réinjecter cette connaissance dans les procédures d'élaboration des cartes d'occupation du sol pour en améliorer la robustesse et la qualité (un des thèmes de recherche de Jordi Inglada au CESBIO).

\subsection{Cartographie de I'humidité du sol: Utilisation d'images radar}

Grâce à l'utilisation croissante des images de télédétection radar, l'OSR dispose désormais de produits tels que des cartes d'humidité du sol avec des précisions meilleures que 5\%. Les premières cartographies effectuées à partir du satellite TERRASAR-X, en mode Stripmap ou Spotlight, permettent pour la première fois de disposer d'un produit d'humidité du sol à très haute résolution spatiale (quelques mètres) et temporelle (11 jours) (Figure 8). L'ajout de cette information biophysique spatialisée est un élément majeur dans l'analyse du paysage agricole et sur les conséquences de gestion du territoire qui en découlent.

A terme, des acquisitions systématiques issues du satellite TERRASAR-X sont envisagées dans le cadre du projet Kalideos développé par le CNES.

\subsection{Cartographie des états de surfaces : Utilisation d'images radar et/ou optique (Approche de fusion de données)}

La synergie entre les capteurs radar et optique permet également d'enrichir la description de la surface. En effet, la fusion d'images FORMOSAT-2 et TERRASAR-X montre un plus grand potentiel (amélioration de 12\%) pour la classification des états de surfaces (sol labouré, sol travaillé, sol semé...) par rapport à leur utilisation indépendante (Figure 9).

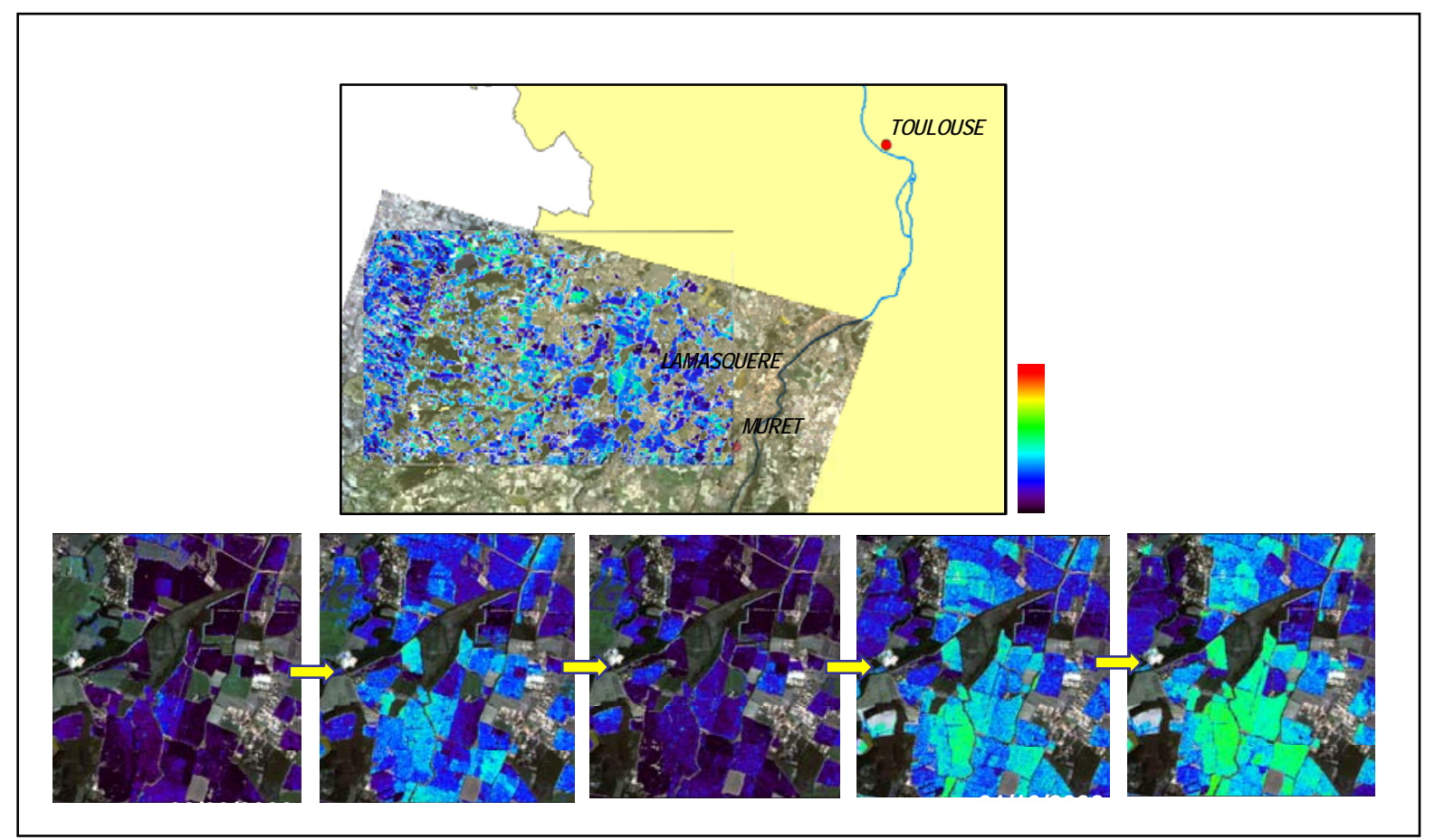

Figure 8. Cartographie de l'humidité du sol nu à partir d'images radar issues du capteur TERRASAR-X. 


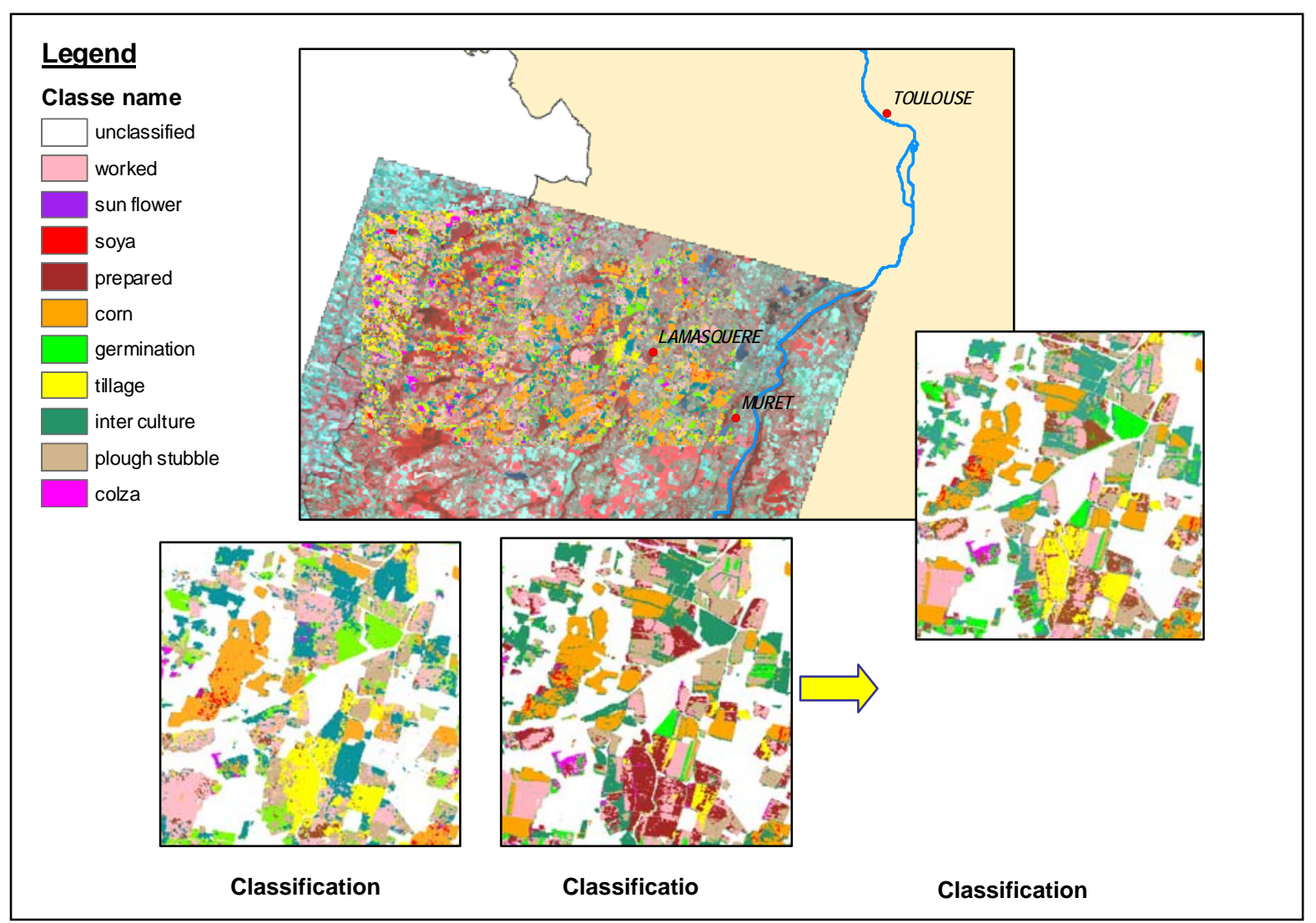

Figure 9 : Classification des états de surface à partir de la fusion d'images optique (FORMOSAT-2) et radar (TERRASAR-X)

\subsection{La modélisation}

La mise en œuvre de programmes de recherche sur le fonctionnement des surfaces continentales aux échelles régionales suppose la définition, la mise en œuvre et l'exploitation de dispositifs d'observation associant mesures et observations in situ, modélisation et données issues de la télédétection spatiale. La dénomination « système d'observation » de l'OSR fait référence à la cohérence globale qui est recherchée au-delà de la diversité de nature des observations. Cette cohérence globale est en grande partie assurée par le recours à la modélisation et par la recherche de synergie entre les activités de recherche sur le fonctionnement des surfaces et le développement des missions spatiales.

Les mesures in-situ sur sites lourdement instrumentés sont utilisées pour la compréhension des processus, leur modélisation à l'aide de modèles existants (e.g. STICS, ISBA) ou en développement (SAFYE, ICARE, ICASTICS), le test de méthodes de télédétection, qu'il s'agisse de l'inversion de variables comme l'indice foliaire ou le couplage télédétection-modélisation. L'acquisition de données sur plusieurs années permet d'échantillonner plusieurs types de cultures et de pratiques ainsi que la variabilité climatique.

Les modèles et méthodes de télédétection mis au point et validés sur les sites expérimentaux et par échantillonnage sur un territoire plus vaste sont utilisés pour obtenir une représentation spatio-temporelle de variables d'intérêt sur l'ensemble du périmètre étudié. Les résultats obtenus jusqu'à présent concernent par exemple l'occupation des terres et l'indice foliaire. L'utilisation des données de télédétection pour piloter des modèles permet d'estimer des variables qui ne sont pas ou difficilement accessibles par télédétection optique, comme par exemple la biomasse, l'humidité du sol ou les flux de chaleur latente. Surtout, l'utilisation des modèles permet d'estimer les variables d'intérêt de manière continue, même lors d'une période sans acquisition d'image. Différentes stratégies sont exploitées pour piloter et contrôler au mieux les modèles à partir d'un échantillon d'observations de télédétection plus ou moins régulier.

A titre d'exemple, les séries temporelles de données SPOT ou FORMOSAT-2 sont utilisées pour étalonner les paramètres du modèle simplifié SAFY (simple algorithm for yield estimates [Duchemin et al., 2008]). SAFY est un modèle paramétrique basé sur l'efficacité d'utilisation de la lumière qui prédit l'indice foliaire (LAI) et la biomasse produite. Les données FORMOSAT-2 ont été utilisées pour étalonner six paramètres de ce modèle, ce qui permet d'aboutir à une estimation spatialisée de la biomasse des cultures tout au long de leur cycle (Figure 10). Les séries temporelles de données de télédétection et leurs produits dérivés, comme le LAI, sont également utilisées pour piloter des modèles plus mécanistes que le modèle SAFY. Les séries temporelles de LAl dérivées de FORMOSAT-2 
ont ainsi été utilisées pour piloter un modèle SVAT décrivant le bilan d'énergie et les échanges de chaleur et de masse entre la surface et l'atmosphère (Figure 11).

Pour l'avenir, une des priorités est d'appliquer les méthodes d'assimilation de données et d'optimisation de paramètres aux modèles couplés qui simulent à la fois la croissance de la végétation, l'humidité du sol, et les échanges entre la surface et l'atmosphère.
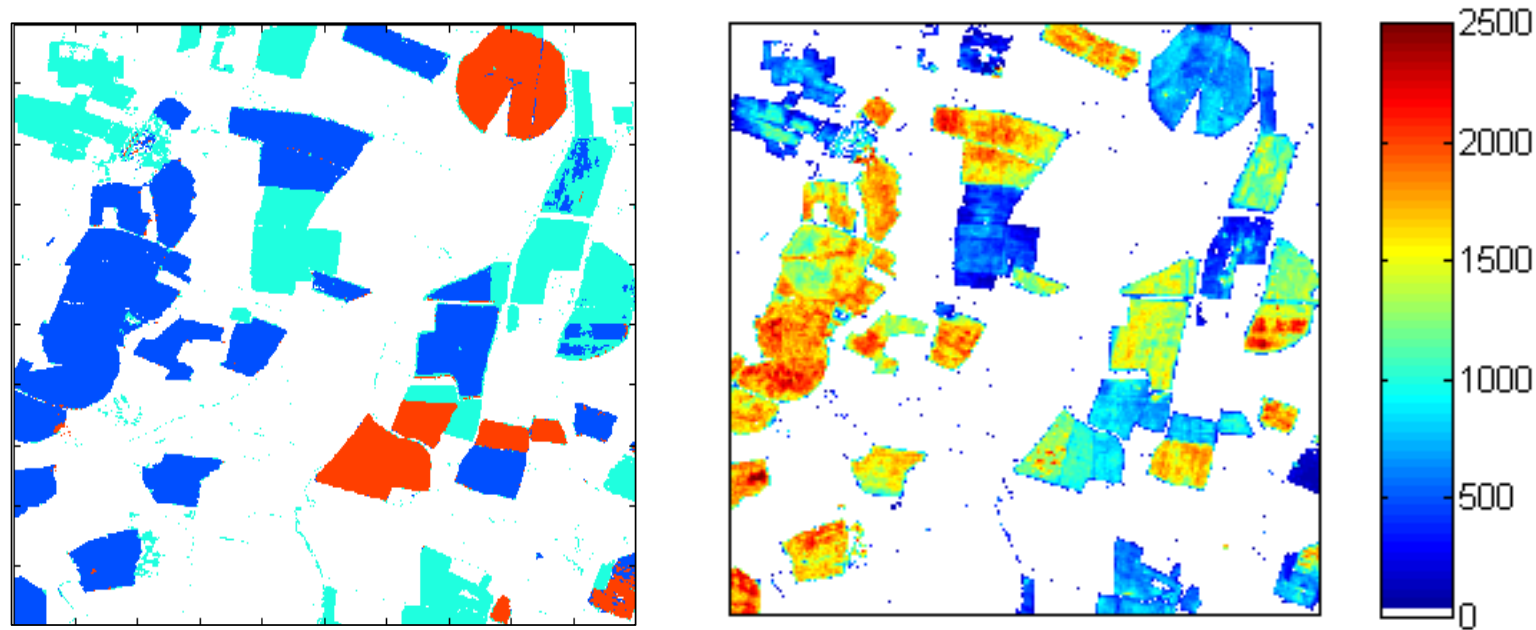

Figure 10 : A gauche : carte des cultures d'été (maïs (bleu), soja (rouge), tournesol (cyan)). A droite, carte des biomasses aériennes estimées à partir d'images FORMOSAT-2 et du modèle SAFY. La zone représentée mesure 500 sur 500 mètres et se situe près de Lamasquère [Claverie et al., soumis].

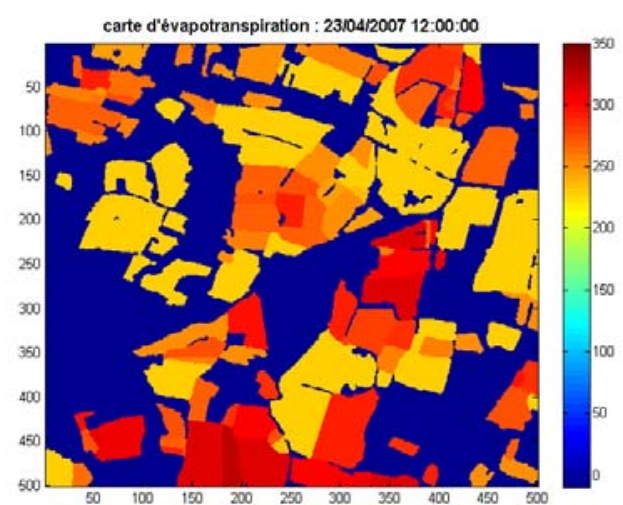

Figure 11 : Exemple de résultat de spatialisation : carte d'évapotranspiration (en watts $/ \mathrm{m}^{2}$ ) obtenue à l'aide d'un SVAT piloté par des données météo et satellitaires de résolution $8 \mathrm{~m}$. La zone, de $4 \times 4 \mathrm{~km}$, est centrée sur le site instrumenté de Lamasquère dont les données ont été utilisées pour valider le SVAT. (Rivalland, communication personnelle)

\section{Perspectives}

Quelques priorités sont d'ores et déjà identifiées concernant les observations et zones suivies. L'extension vers le sud de la zone OSR MiPy, en direction des Pyrénées, sur au moins $30 \mathrm{~km}$, est hautement souhaitable de par les gradients climatiques qu'elle offre et pour la prise en compte de problématiques spécifiques à la montagne, complémentaires de celles de la zone actuelle de coteaux et vallées. Un transect nord-sud est demandé pour la mission VEN $\mu \mathrm{S}$ et 45 images d'archives SPOT
2001-2011 viennent d'être commandées par le CESBIO et 6 autres partenaires pour couvrir le Luchonais et l'est du parc naturel régional des Pyrénées Ariégeoises. Via un autre projet (Tosca), c'est l'ensemble de la région Midi-Pyrénées qui va être pris en compte avec des archives multi-temporelles SPOT 10 mètres en 2008 et 2009, pour tester et développer des méthodes opérationnelles d'élaboration de cartes d'occupation du sol dans un contexte GMES-Sentinel-2.

La démarche d'observatoire du comportement de la végétation en réponse à des conditions de milieux, de 
pratiques et d'années climatiques particulières semble prometteuse. Elle est une étape clé dans une démarche globale et itérative, en amont et en aval des étapes de classifications d'occupation du sol et de modélisation. Les pistes actuelles sont de coupler suivis en basse et haute résolution spatiale et d'intégrer l'analyse du milieu dans les diagnostics effectués : effets des sols, réserves utiles, expositions et altitudes, indices topographiques.

Pour l'élaboration des cartographies d'occupation du sol, les principaux enjeux méthodologiques des travaux en cours portent sur l'amélioration forte de la qualité des résultats, de la fréquence d'actualisation, l'enrichissement de la nomenclature et l'automatisation. La voie privilégiée repose sur l'utilisation combinée des dimensions spectrale (solaire, infrarouge thermique et radar) et temporelle (répétitivité élevée), et l'association des mesures de télédétection à des données exogènes au moyen de systèmes experts (règles de décision à dire d'expert et basées sur des modèles de processus).

Les pistes sont nombreuses aussi pour la modélisation et l'usage des modèles. Un des aspects majeur des travaux va porter sur la combinaison de l'optique et du radar pour caractériser les états de surface, et globalement la modélisation intégrée en lien avec les aspects hydriques (humidité des sols, biomasses, besoin en eau...).

Si le CESBIO a été le concepteur et le principal utilisateur de l'OSR, de nombreux partenaires scientifiques et socio-économiques s'impliquent maintenant de plus en plus fortement dans les activités KALIDEOS OSR MIPY. Cela devrait être facilité par plusieurs projets et dispositifs structurants, pour la recherche (Observatoire Pyrénéen du Changement Climatique...) ou pour les applications (Pôle d'Expérimentation et d'Applications des Technologies Satellitaires, Territoires Démonstrateurs de Services).

\section{Remerciements}

Le dispositif OSR est principalement soutenu par le CNRS-INSU, l'Université Paul Sabatier, la Région MidiPyrénées et le CNES. Les auteurs souhaitent remercier le CNES (Centre National d'Études Spatiales) pour l'accès gratuit aux données SPOT et FORMOSAT-2 par le biais du programme KALIDEOS (http://kalideos.cnes.fr). De nombreux collègues du CESBIO sont aussi remerciés pour leur contribution aux mesures de terrain et aux travaux (Hervé Gibrin, Nicole Ferroni, Pascal Keravec, Bernard Marciel, Jordi Inglada, Vincent Rivalland, Marcela Arias, Mehdi Lefeuvre, Martin Claverie, Rémy Fieuzal, Antoine Masse, ...).

\section{Références}

Auda Y., Dejoux J.-F., Ducrot D., Gouaux P., Hagolle O., Le Page M., Suere C., and Dedieu G., 1996. Utilisation d'un SIG nomade couplé à un GPS pour cartographier les paysages du Sud-Ouest toulousain. XYZ n 118, pp 47-50.

Beziat P., Ceschia E., Dedieu G., 2009. Carbon balance of a three crop succession over two cropland sites in South West France. Agricultural and Forest Meteorology, 149(10), pp 1628-1645.

Cabot, F., Hagolle, O., Ruffel, C., Henry, P.J., 1999. Remote sensing data respository for in-flight calibration of optical sensors over terrestrial targets. In: Earth Observing Systems IV. Presented at the Earth Observing Systems IV, SPIE, Denver, CO, USA, pp. 514-523.

Ceschia E., Béziat P., Dejoux J.F, Aubinet M., Bernhofer Ch., Bodson B., Buchmann N., et al., 2010. Management effects on net ecosystem carbon and GHG budgets at European crop sites Special Issue: C Balance Euro-Croplands. Agriculture, Ecosystems and Environment, $\mathrm{n}^{\circ} 139$, pp 363383.

Chéret, V., J.P. Denux, M. Gay, P. Thévenet, and C. Gacherieu, 2008. Utilisation de séries temporelles d'images satellitales pour cartographier le dépérissement des boisements résineux du Sud Massif Central. Journées des applications spatiales, CNES, 22-25 avril 2008, Toulouse.

Claverie M., Demarez V., Duchemin B., Hagolle O., Ducrot D., Marais-Sicre C., Dejoux J.F., Huc M., Keravec P., Béziat P., Fieuzal R., Ceschia E. and Dedieu G. Crop biomass estimation using high spatial and temporal resolution remote sensing data. Remote Sensing of Environment, Soumis.

Coret L, Maisongrande P, Boone A, Lobo A, Dedieu G, Gouaux P., 2005. Documentation of the Drought impact with SPOT/HRVIR images tile Series over South-Western France. International Journal of Remote Sensing, $\mathrm{n}^{\circ}$ 26, pp 2461-2469.

Dedieu, G., Karnieli, A., Hagolle, O., Jeanjean, H., Cabot, F., Ferrier, P., Yaniv, Y., 2006. VEN $\mu$ S : A joint Israel-French Earth Observation scientific mission with High spatial and temporal resolution capabilities. In: Recent Advances in Quantitative Remote Sensing., J.A.Sobrino, Valencia, Spain, pp. 517-521.

Demarez V., Duthoit S., Baret F. Weiss M., Dedieu G., 2007. Estimation of leaf area and clumping indexes of crops with hemispherical photographs, Agric. Forest Meteorol., Vol 148/4, pp 644-655.

Duchemin, B., Maisongrande, P., Boulet, G., Benhadj, I., 2008. A simple algorithm for yield estimates: Evaluation for semi-arid irrigated winter wheat monitored with green leaf area index. Environmental Modelling \& Software, $n^{\circ} 23, p p$ 876-892.

Ducrot D., Masse A., Marais-Sicre C., Dejoux J.-F., Baup F., 2010. Multisensor and multitemporal image fusion methods to improve remote sensing image classification. In: Recent Advances in Quantitative Remote Sensing, J.A.Sobrino, Valencia, Spain,

Hagolle O., Dedieu G., Mougenot B., Debaecker V., Duchemin B. and Meygret A., 2008. Correction of aerosol effects on multi-temporal images acquired with constant viewing angles: Application to FORMOSAT-2 images. Remote sensing Environment, Vol. 112, Issue 4, pp 16891701. 
Hagolle O., Huc M., Villa Pascual D., and Dedieu G., 2010. A multi-temporal method for cloud detection, applied to FORMOSAT-2, VEN $\mu$ S, LANDSAT and SENTINEL-2 images. Remote Sensing of Environment, vol. 114, pp. 1747-1755.

Lafrance B., Lenot X., Ruffel C., Cao P., Rabaute T., 2011. KALIDEOS - Outils de prétraitements des images optiques. Revue française de photogrammétrie et de télédétection.

Masse A., Ducrot D., Marais-Sicre C., Dejoux J.-F, Baup F. Novel fusion methods to improve multi-source remote sensing image classification. IJRS (en révision). 